\title{
Repeatability and reproducibility of measurements of low dissolved radiocesium concentrations in freshwater using different pre-concentration methods
}

\author{
Momo Kurihara ${ }^{1,24} \cdot$ Tetsuo Yasutaka $^{1}$ Tatsuo Aono ${ }^{2} \cdot$ Nobuo Ashikawa $^{3} \cdot$ Hiroyuki Ebina $^{4} \cdot$ Takeshi lijima $^{5}$. \\ Kei Ishimaru ${ }^{6} \cdot$ Ramon Kanai $^{7} \cdot$ Zin'ichi Karube $^{8,25}$ - Yae Konnai ${ }^{9}$. Tomijiro Kubota ${ }^{10}$ - Yuji Maehara ${ }^{11}$. \\ Takeshi Maeyama ${ }^{12}$ - Yusuke Okizawa ${ }^{13}$. Hiroaki Ota ${ }^{14} \cdot$ Shigeyoshi Otosaka $^{15,26} \cdot$ Aya Sakaguchi $^{16}$. \\ Hisaya Tagomori ${ }^{17} \cdot$ Keisuke Taniguchi $^{18} \cdot$ Masatoshi Tomita $^{19} \cdot$ Hirofumi Tsukada $^{20} \cdot$ Seiji Hayashi $^{21}$. \\ Sangyoon Lee Le,27 $_{\text {S Susumu Miyazu }}{ }^{10,28} \cdot$ Moono Shin ${ }^{22,29} \cdot$ Takahiro Nakanishi $^{23} \cdot$ Tatsuhiro Nishikiori $^{22}$. \\ Yuichi Onda ${ }^{16} \cdot$ Takuro Shinano $^{22,30} \cdot$ Hideki Tsuji $^{21}$
}

Received: 5 May 2019 / Published online: 16 August 2019

(c) The Author(s) 2019

\begin{abstract}
We assessed the repeatability and reproducibility of methods for determining low dissolved radiocesium concentrations in freshwater in Fukushima. Twenty-one laboratories pre-concentrated three of $10 \mathrm{~L}$ samples by five different pre-concentration methods (Prussian-blue-impregnated filter cartridges, coprecipitation with ammonium phosphomolybdate, evaporation, solid-phase extraction disks, and ion-exchange resin columns), and activity of radiocesium was measured. The $z$-scores for all of the ${ }^{137} \mathrm{Cs}$ results were within \pm 2 , indicating that the methods were a good degree of precision. The relative standard deviations (RSDs) indicating the reproducibility among different laboratories were larger than the RSDs indicating the repeatability in each separate laboratory.
\end{abstract}

Keywords Measurement precision $\cdot$ Fukushima $\cdot$ River water $\cdot{ }^{137} \mathrm{Cs} \cdot{ }^{134} \mathrm{Cs} \cdot \mathrm{Ge}$-detector

\section{Introduction}

It has been necessary to measure dissolved radiocesium $\left({ }^{134} \mathrm{Cs}\right.$ and $\left.{ }^{137} \mathrm{Cs}\right)$ concentrations in freshwater near the Fukushima Daiichi Nuclear Power Plant (FDNPP) since the FDNPP accident to provide information to allow root uptake by crops and accumulation in aquatic organisms to be assessed. Many institutions have therefore monitored dissolved radiocesium concentrations in freshwater in northeastern Japan since the FDNPP accident [1-12].

Dissolved and particulate-bound radiocesium concentrations in freshwater in the northeastern Japan are mostly $<0.1 \mathrm{~Bq} \mathrm{~L}^{-1}$, and dissolved radiocesium concentrations

Electronic supplementary material The online version of this article (https://doi.org/10.1007/s10967-019-06696-2) contains supplementary material, which is available to authorized users.

Tetsuo Yasutaka

t.yasutaka@aist.go.jp

Extended author information available on the last page of the article of $0.001-0.1 \mathrm{~Bq} \mathrm{~L}^{-1}$ have been found at most monitoring points in the northeastern Japan [9, 12-15]. The low concentrations mean that effective quantitative measurements of dissolved radiocesium in freshwater require samples to be pre-concentrated before analysis. Environmental radioactivity measurement manuals published by the Japanese Ministry of Education, Culture, Sports, Science and Technology contain methods for measuring dissolved radiocesium in water involving either evaporating samples to dryness or ion exchange [16]. Other methods involve ammonium phosphomolybdate (AMP) coprecipitation, particularly for very saline samples, and instrumental analysis using a germanium semiconductor detector (Ge-detector) [17]. These methods have been used to quantitatively determine dissolved radiocesium in freshwater for a long time (since before the FDNPP accident). New pre-concentration methods were developed after the FDNPP accident, including using cartridges impregnated with Prussian blue (PB) [18-21] and solid-phase extraction disks [22, 23], to decrease the time required for pre-concentration and solid-liquid separation. These new concentration methods dramatically decreased 
the time required for analyzing samples, so have been used widely.

The precision achieved when analyzing radiocesium using different methods has been assessed previously. Five methods (involving evaporation to dryness, AMP coprecipitation, PB cartridges, solid-phase disks, and ion exchange resin) were used to analyze three water samples (two ionexchanged water samples spiked with radiocesium and one freshwater sample) containing dissolved radiocesium at concentrations of $0.01-1.0 \mathrm{~Bq} \mathrm{~L}^{-1}$ by 18 laboratories [24]. The relative standard deviations (RSDs) were 0.08-0.119, and the $z$-scores for $>80 \%$ of the results were within \pm 2 . The results indicated that the pre-concentration methods (including the new methods) are good degree of precision. However, only one sample was analyzed repeatedly, so the results did not indicate whether the slight variations in the concentrations were caused predominantly by variability in the results from each laboratory or by variability between the results from different laboratories. The results also did not indicate whether variability between the results from different laboratories may have been caused by different pre-concentration methods being used by the different laboratories.

In the study presented here, we evaluated the variability in dissolved ${ }^{134} \mathrm{Cs}$ and ${ }^{137} \mathrm{Cs}$ concentrations in freshwater samples determined by each participating laboratory (repeatability) and variability in the concentrations determined by the different laboratories (reproducibility). Each participating laboratory analyzed three samples under the same conditions, then all the samples were analyzed in one laboratory to remove the effect of using different instruments at different laboratories on the reproducibility of the results. We assessed the repeatability and reproducibility and the factors affecting the reproducibility of the results from different laboratories.

\section{Materials and methods}

\section{Water sample collection}

River water was collected from the Abukuma Kogen region in Japan on 25 October 2017 under base flow condition. Most radiocesium in the sample was supplied by the FDNPP accident in March 2011. Approximately $1200 \mathrm{~L}$ of river water was collected in six $200 \mathrm{~L}$ tanks. The water was pumped from the river at the center of a bridge. The water was immediately transported to the laboratory, where it was passed through A045A $0.45 \mu \mathrm{m}$ membrane filters (Advantec MFS, Dublin, CA, USA) on 26 October 2017. The filtered water in a $1200 \mathrm{~L}$ tank was stirred by passing it through a pump at a flow rate of $3 \mathrm{~L} \mathrm{~s}^{-1}$ for $>12 \mathrm{~h}$. On 27 October 2017, $50 \mathrm{~L}$ of well-mixed water was removed using a washed plastic bucket, and three $10 \mathrm{~L}$ polyethylene flexible tanks were filled with the water. The flexible tanks were then refrigerated until they were sent to a participating laboratory. This was repeated for each participating laboratory. The samples were sent to the participating laboratories on 7 November 2017. Each partitioning laboratory received its allocation of three $10 \mathrm{~L}$ samples on 8 November 2017 and immediately stored the samples in a refrigerator. We indicated the procedure for sample storage keeping in a refrigerator but did not indicate the acidic treatment because of limiting condition of pre-treatment method. Notable procedures for sample storage for each laboratory are shown in Table S1.

The mean \pm standard deviation $\mathrm{Na}^{+}$concentrations, $\mathrm{Ca}^{2+}$ concentrations, $\mathrm{pH}$ values, and electrical conductivities for the $10 \mathrm{~L}$ samples were $3.5 \pm 0.06 \mathrm{mg} \mathrm{L}^{-1}, 2.3 \pm 0.06 \mathrm{mg} \mathrm{L}^{-1}$, $7.2 \pm 0.05$, and $3.7 \pm 0.05 \mathrm{mS} \mathrm{m}^{-1}$, respectively. We concluded that all the samples sent to the participating laboratories were well mixed because similar $\mathrm{Na}^{+}$concentrations, $\mathrm{Ca}^{2+}$ concentrations, $\mathrm{pH}$ values, and electrical conductivities were found by the different laboratories.

\section{Pre-concentration methods and radioactivity measurements}

Five pre-concentration methods (PB filter cartridges, AMP coprecipitation, evaporation, solid-phase extraction disks, and ion-exchange resin) were used by the partitioning laboratories (Fig. 1). The test involved ${ }^{134} \mathrm{Cs}$ and ${ }^{137} \mathrm{Cs}$ concentrations in 68 water subsamples being determined by 21 Japanese laboratories. Each laboratory except one pre-concentrated and determined the radioactivity in three water samples (one laboratory only analyzed two samples, see Table 1). Two laboratories analyzed six water subsamples using two concentration methods, and these laboratories were each given two laboratory ID numbers.

The five pre-concentration methods are described below. The PB filter cartridge method takes advantage of $\mathrm{PB}$ $\left(\mathrm{KFe}\left[\mathrm{Fe}(\mathrm{CN})_{6}\right]_{3} \cdot \mathrm{xH}_{2} \mathrm{O}\right)$ being able to adsorb dissolved radiocesium with a good degree of specificity [18-21]. The PB filter cartridge method involved removing dissolved radiocesium from water passed through a nonwoven fabric impregnated with PB in a cartridge. A water sample was passed through a cartridge at a flow rate of $2.5 \mathrm{~L} \mathrm{~min}^{-1}$ by pumping, and ${ }^{134} \mathrm{Cs}$ and ${ }^{137} \mathrm{Cs}$ radioactivity in each cartridge was then measured using a Ge-detector with a special cartridge container. As distribution of radiocesium in the cartridge is not uniform, geometric correction was performed to evaluate concentration [21]. Seven laboratories used potassium zinc ferrocyanide PB cartridges [21], and one laboratory used copper-substituted PB cartridges [25].

The AMP coprecipitation method takes advantage of the ability of AMP to adsorb dissolved radiocesium with a good degree of specificity [17]. Each water sample was adjusted 
Fig. 1 Five pre-concentration methods (PB filter cartridges, AMP coprecipitation, evaporation, solid-phase extraction disks, and ion-exchange resin) used by the partitioning laboratories

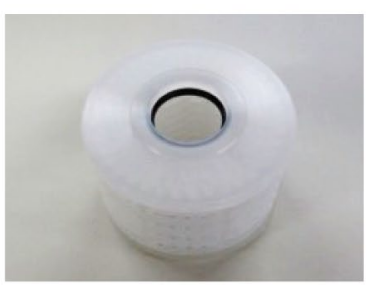

PB filter cartridge

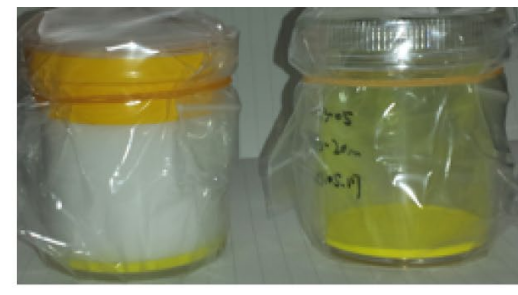

AMP coprecipitation

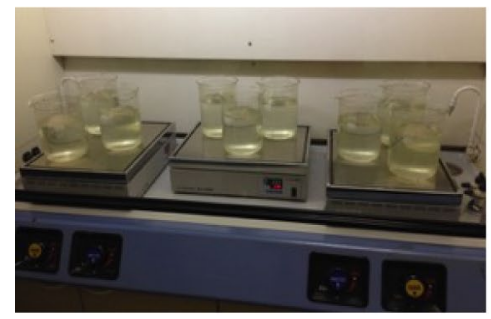

Evaporation

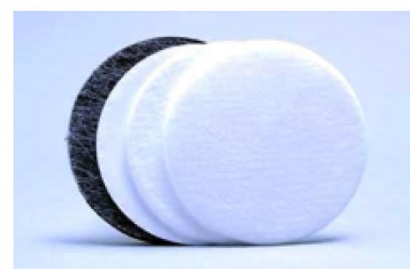

Solid-phase extraction disk

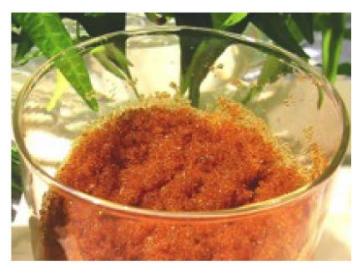

Ion-exchange resin
Table 1 Number of laboratories using each pre-concentration method and the number of samples analyzed using each pre-concentration method

\begin{tabular}{lcc}
\hline Pre-concentration method & No. of laboratories & $\begin{array}{l}\text { No. of } \\
\text { samples }\end{array}$ \\
\hline Prussian blue filter cartridge & 8 & 24 \\
Ammonium phosphomolybdate & 6 & 18 \\
$\quad$ coprecipitation & 5 & 15 \\
Evaporation & 3 & 9 \\
Solid-phase extraction disk & 1 & 2 \\
Ion exchange resin & 23 & 68 \\
Total & & \\
\hline
\end{tabular}

${ }^{\mathrm{a}}$ The total number of laboratories is not the same as the number of participating laboratories

to $\sim \mathrm{pH} 1.5$ before analysis. The radiocesium in the sample was quantitatively removed from the water sample by coprecipitation as AMP/Cs compounds once AMP had been added. The AMP/Cs compounds were removed by filtering the sample, then dried and transferred to a small container suitable for radioactivity measurement (e.g. $100 \mathrm{ml}$ with 5 -cm diameter plastic container) before the radioactivity was measured using a $\mathrm{Ge}$-detector.

The evaporation method is to measure concentrated radiocesium in a water sample by evaporation [16]. In the evaporation method in the present test, a water sample was evaporated in a glass beaker on a hot plate but not boiled excessively. Each sample was evaporated to a volume of between $50 \mathrm{~mL}$ and $2 \mathrm{~L}$, then transferred to a $100 \mathrm{~mL}$ container or a $2 \mathrm{~L}$ Marinelli beaker before the radioactivity was measured using a Ge-detector. One laboratory acidified the samples before the evaporation method was performed.
In the solid-phase extraction disk method, dissolved radiocesium was removed from a water sample passed through a solid-phase extraction disk containing ion-selective resin on a Teflon support $[26,27]$. A water sample was passed through a disk using a pressure filtration system, and the ${ }^{134} \mathrm{Cs}$ and ${ }^{137} \mathrm{Cs}$ radioactivity in the disk was measured using a Ge-detector.

In the ion exchange resin method, dissolved radiocesium is collected to a cation exchange resin from a water sample [16]. The ion exchange resin method in the present test was performed by passing a water sample through a $100 \mathrm{~mL}$ column containing a cation exchange resin in the $\mathrm{Na}^{+}$form. The resin containing the radiocesium was then transferred to a $100 \mathrm{~mL}$ container and the radioactivity was measured using a Ge-detector.

The more detailed information of each pre-concentration methods have been described previously [21, 22, 24, 28, 29]. The specific differences in procedures among laboratories are summarized in Table $\mathrm{S} 1$.

The laboratories pre-concentrated the samples between 8 and 25 November 2017 and determined the radioactivity of ${ }^{134} \mathrm{Cs}$ and ${ }^{137} \mathrm{Cs}$ in the samples by 10 January 2018 . The radioactivity measurements were made in the laboratories by using their own Ge-detectors. Each laboratory followed its usual operating procedures for determination of low dissolved radiocesium concentrations in freshwater (e.g., sample storage procedure and measurement time). Radioactivity of ${ }^{134} \mathrm{Cs}$ and ${ }^{137} \mathrm{Cs}$ were decay corrected to 12:00 JST 11 November 2017 to allow the results to be compared. The different pre-concentration methods gave samples with different shapes, so each laboratory calibrated its detector(s) using a standard radioactive source (a special customized source was used for each sample of the same type). Finally, in order to discriminate effects of radioactivity measurement 
by different detectors from different procedures for sample treatment among laboratories, the samples were sent to one laboratory (Tokyo Power Technology Ltd., Chiba, Japan), where the ${ }^{137} \mathrm{Cs}$ radioactivity in each sample was measured using a single Ge-detector (using a 43,000 s measurement period).

\section{Statistical analysis}

The ${ }^{134} \mathrm{Cs}$ and ${ }^{137} \mathrm{Cs}$ concentrations found in the water samples were evaluated using $z$-scores, as described in a previous publication [30] and in the IAEA-JAPAN proficiency test [31]. Each $z$-score was calculated using the equation

$Z=\left(x_{i}-X\right) / s$,

where $x_{i}$ is the concentration in water sample $i, X$ is the robust average, and $s$ is the robust standard deviation. The robust average and robust standard deviation were calculated using algorithm A1, from JIS Z 8405 [32].
The repeatability and reproducibility were assessed using the relative standard deviation (RSD), as described in a previous publication [30]. Each RSD was calculated using the equation

$\mathrm{RSD}=s / X$.

The repeatability (indicating the precision of repeated analyses) for each laboratory was assessed using the RSD for that laboratory $(n=3)$. The reproducibility (indicating variability between the results from different laboratories) was assessed using the RSD calculated from the robust average concentrations produced by the laboratories, as shown in Fig. 2.
Fig. 2 Scheme used to determine the repeatability and reproducibility relative standard deviations (RSD)

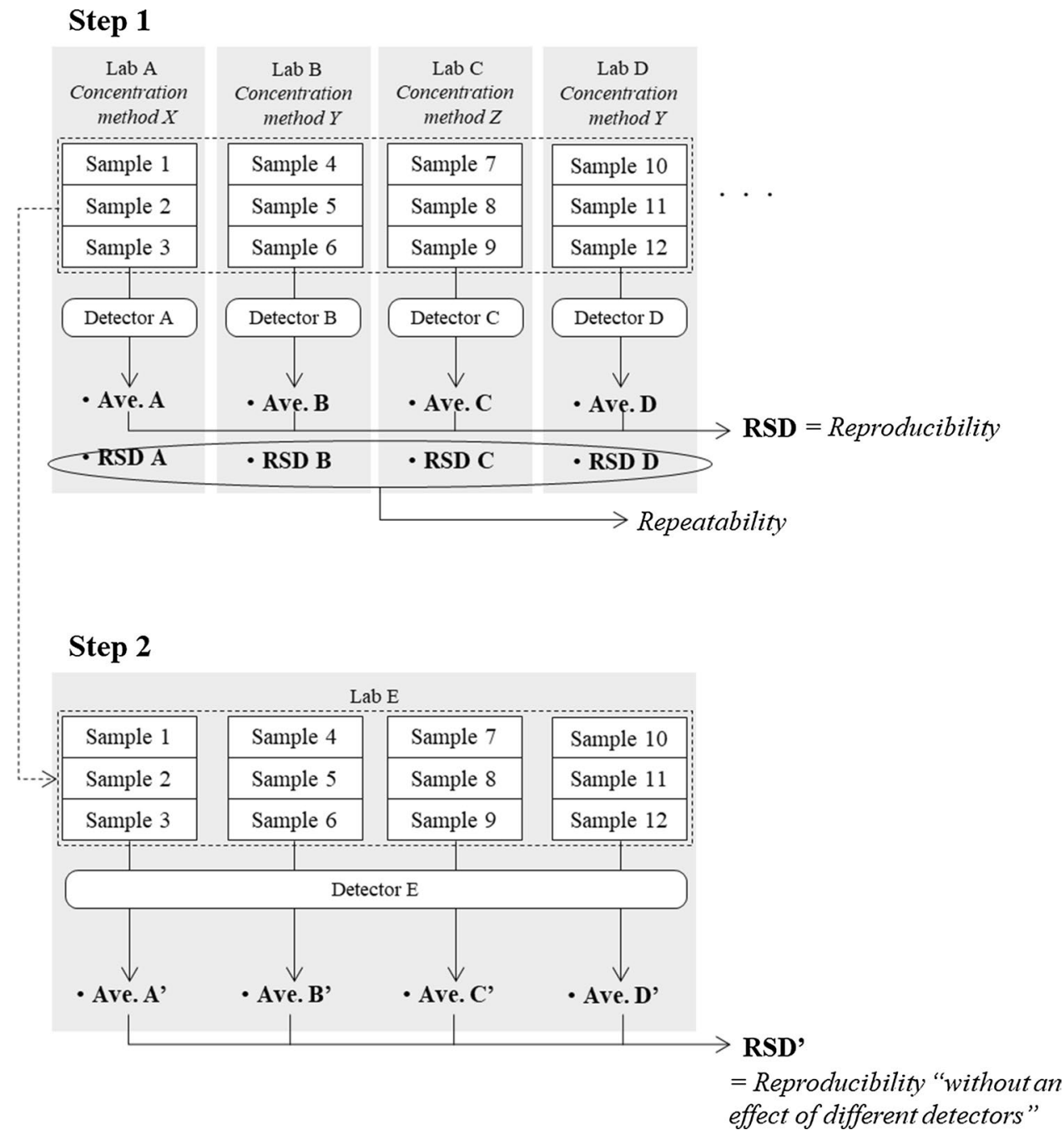




\section{Results and discussion}

\section{Assessment of the precision of the results}

The robust averages \pm the standard deviations of the ${ }^{134} \mathrm{Cs}$ and ${ }^{137} \mathrm{Cs}$ concentrations determined by the laboratories were $0.020 \pm 0.002$ and $0.153 \pm 0.013 \mathrm{~Bq} \mathrm{~L}^{-1}$, respectively. The $z$-scores were within \pm 2 . The ${ }^{134} \mathrm{Cs}$ and ${ }^{137} \mathrm{Cs}$ concentrations were $0.015-0.025$ and $0.131-0.169 \mathrm{~Bq} \mathrm{~L}^{-1}$, respectively, and the results produced by the different laboratories were not markedly different. The $z$-scores for $>95 \%$ of the ${ }^{134} \mathrm{Cs}$ results and all of the ${ }^{137} \mathrm{Cs}$ results were within \pm 2 (Fig. 3a, b), suggesting that there was good degree of reproducibility between the methods used, as has been found previously [24].

The robust average ${ }^{134} \mathrm{Cs}$ and ${ }^{137} \mathrm{Cs}$ concentrations determined using the five different pre-concentration methods were not markedly different (Fig. 4a, b). The ${ }^{134} \mathrm{Cs}$ and ${ }^{137} \mathrm{Cs}$ concentrations determined using the PB cartridge, AMP coprecipitation, evaporation, and solid-phase disk methods did not show statistically significant difference (one-way ANOVA, $p<0.005$, the concentrations determined using the ion-exchange resin method was not included in the analysis because only one laboratory used for the determination). This indicated that differences in the concentrations found by the different laboratories were not caused by the laboratories using different pre-concentration methods. The concentrations found using the different pre-concentration methods
Fig. 3 Robust average $\mathbf{a}^{134} \mathrm{Cs}$ concentrations and $\mathbf{b}{ }^{137} \mathrm{Cs}$ concentrations from each laboratory in Step 1, and $\mathbf{c}^{137} \mathrm{Cs}$ concentrations at laboratory in Step 2. The error bars indicate the standard deviations

\begin{tabular}{|c|c|c|}
\hline$\triangle \mathrm{PB}$ cartridge & Evaporation & $\square$ Ion-exchange \\
\hline$\diamond \mathrm{AMP}$ & Solid-phase disk & \\
\hline
\end{tabular}
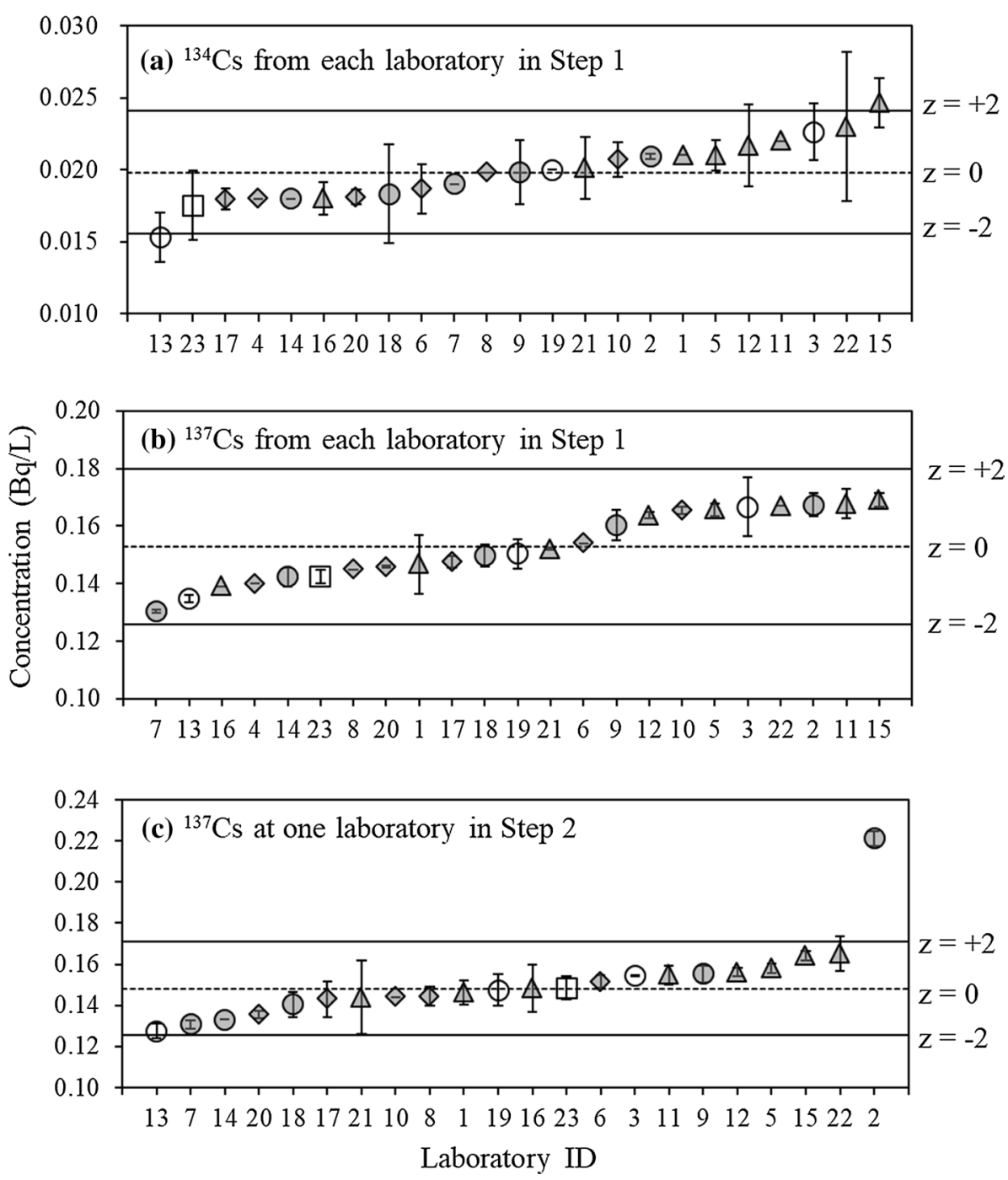

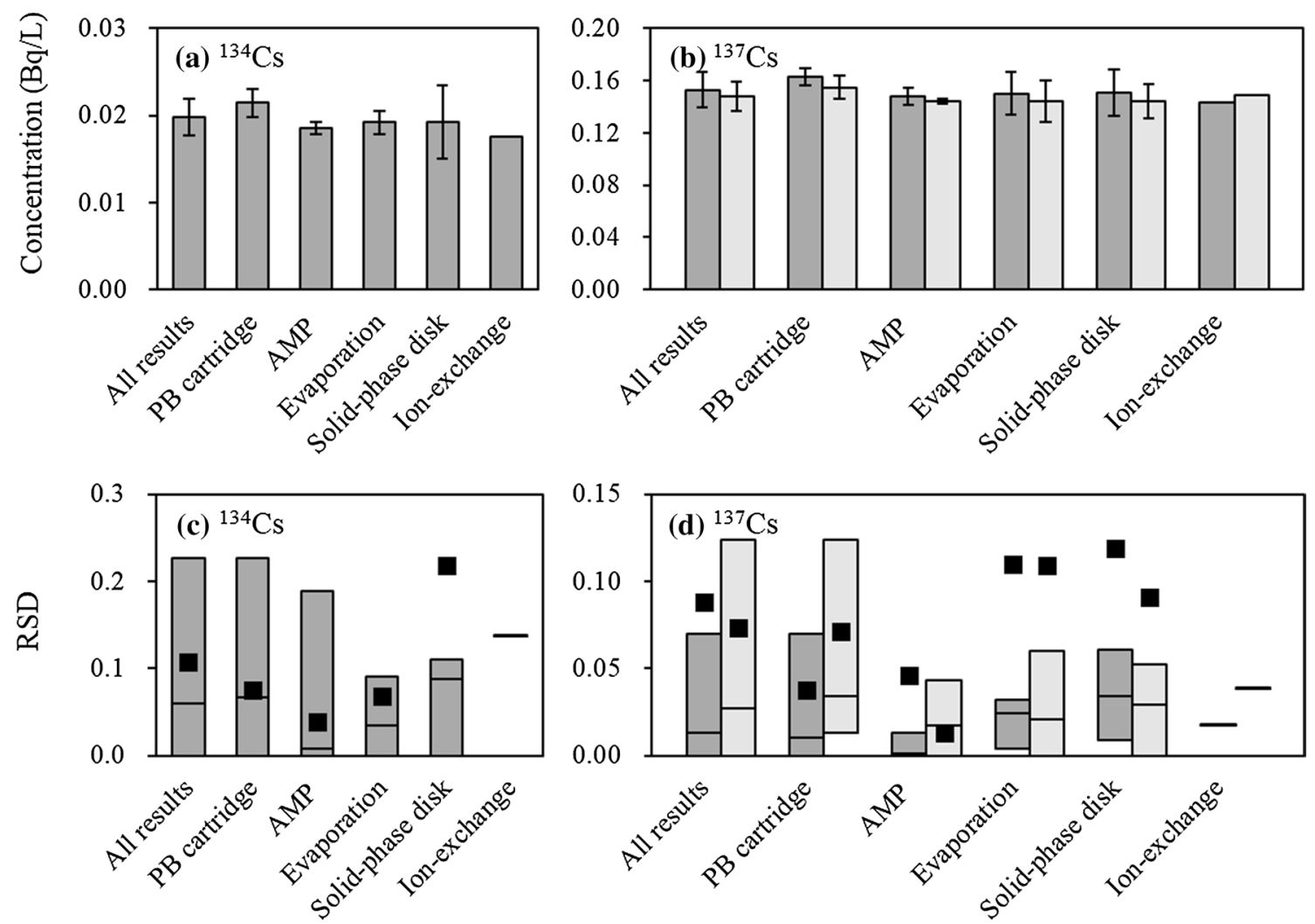

From each laboratory in Step 1

- $\longrightarrow$ Reproducibility RSD

At one laboratory in Step 2

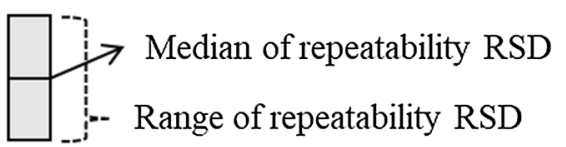

Fig. 4 Robust average a ${ }^{134} \mathrm{Cs}$ concentrations and $\mathbf{b}{ }^{137} \mathrm{Cs}$ concentrations for the different pre-concentration methods. The error bars indicate the standard deviations. Comparison between repeatability and reproducibility relative standard deviations (RSDs) for $\mathbf{c}^{134} \mathrm{Cs}$ con-

were not statistically significantly different, but the ${ }^{134} \mathrm{Cs}$ and ${ }^{137} \mathrm{Cs}$ concentrations were higher when the $\mathrm{PB}$ cartridge method was used (at 0.021 and $0.163 \mathrm{~Bq} \mathrm{~L}^{-1}$, respectively) than when the other pre-concentration methods were used. Similar results were found in the previous multi-laboratory evaluation [24]. However, the participating laboratories did not perform the entire analytical processes following exactly the same procedures even when the same pre-concentration methods were used. We cannot therefore conclude that the different radiocesium concentrations found by the different laboratories were caused by different pre-concentration methods being used. centrations and $\mathbf{d}{ }^{137} \mathrm{Cs}$ concentrations for the different pre-concentration methods. Bar charts indicate range of repeatability RSDs and middle lines of the bars are medians. Solid square symbols indicate reproducibility RSD

\section{Repeatability RSD}

The ranges and median repeatability RSDs for the participating laboratories are shown in bar charts in Fig. 4c, d. The repeatability for the laboratories can be compared from the figure. For all the results, the ${ }^{134} \mathrm{Cs}$ repeatability RSDs were $0-0.226$ (median 0.059 ), and the ${ }^{137}$ Cs repeatability RSDs were $0-0.070$ (median 0.013 ). The repeatability RSDs were different for the different laboratories, suggesting that repeatability was different for different laboratories. Like for the concentrations, the repeatability RSDs for four of the pre-concentration methods did not show significantly statistically difference (one-way ANOVA, $p<0.005$, the concentrations determined using the ion-exchange resin method 
was not included in the analysis because only one laboratory used for the determination). The repeatability RSDs for the laboratories using the AMP method (median 0.008 for ${ }^{134} \mathrm{Cs}$ and median 0.001 for ${ }^{137} \mathrm{Cs}$ ) were smaller (but not statistically significantly smaller) than the repeatability RSDs for the laboratories using the other pre-concentration methods. This may have been because the laboratories using the AMP method have used it for a long time (since before the FDNPP accident), meaning they have been able to minimize variability between repeat analyses [24].

\section{Reproducibility RSD and analyses of all the samples by one laboratory}

The reproducibility RSDs for each pre-concentration method and for the results for all the pre-concentration methods combined are shown in solid square symbols in Fig. 4c, d. The reproducibility RSDs indicate variability between the results produced by the different laboratories. The reproducibility RSDs for all the ${ }^{134} \mathrm{Cs}$ and ${ }^{137} \mathrm{Cs}$ results were 0.108 and 0.088 , respectively. The median ${ }^{134} \mathrm{Cs}$ and ${ }^{137} \mathrm{Cs}$ reproducibility RSDs were 0.059 and 0.013 , respectively, indicating that variability between laboratories was generally larger than variability within a laboratory. The reproducibility RSDs for the different pre-concentration methods were not markedly different, but the reproducibility RSDs were lower for the AMP method than for the other methods. This indicated that the methods offered good reproducibility between laboratories as well as within each laboratory.

The results shown above do not describe the separate effects of different pre-concentration methods, Ge-detectors, and other factors on reproducibility. Comparisons of ${ }^{137} \mathrm{Cs}$ concentration and RSD between from each laboratory and at one laboratory are shown in Figs. $3 c$ and $4 b$, d. The robust average ${ }^{137} \mathrm{Cs}$ concentration \pm the standard deviation was $0.145 \pm 0.011 \mathrm{~Bq} \mathrm{~L}^{-1}$ and the reproducibility RSD was 0.073 . The average was not different from the averages for the different laboratories. The ${ }^{137} \mathrm{Cs}$ concentrations for the PB cartridge, AMP coprecipitation, evaporation, and solidphase disk methods were not statistically significantly different (one-way ANOVA, $p<0.005$ ).

The robust averages and standard deviations for the ${ }^{137} \mathrm{Cs}$ concentrations measured at a single laboratory are shown in Fig. 2c. The average concentrations were similar to the concentrations by each laboratory shown in Fig. $2 b$ except for laboratory 2 . The reproducibility RSDs measured at a single laboratory were almost the same as the reproducibility RSDs for the different laboratories (Fig. 3d). This suggests that the differences in the results from the different laboratories were not predominantly caused by the different pre-concentration methods and different Ge-detectors used but rather by other factors. The $z$-scores for $>90 \%$ of the ${ }^{137} \mathrm{Cs}$ results were within \pm 2 , suggesting that the pre-concentration methods used by the laboratories all offered a good degree of precision.

\section{Conclusions}

The measurement precision with which low dissolved radiocesium concentrations in freshwater samples were determined by 21 Japanese laboratories was assessed. The repeatability and reproducibility of the results were assessed. All the pre-concentration methods used by the different laboratories gave a good degree of precision, indicating that even techniques used by Japanese laboratories to quantitatively determine low dissolved radiocesium concentrations only since the FDNPP accident in 2011 were high precision. Variability in the concentrations found by different laboratories was larger than variability in the concentrations found by each laboratory. Variability between laboratories was probably not caused by different pre-concentration methods or Ge-detectors being used but by other differences in the sample treatment procedures. This should be investigated further. Few evaluations of the measurement precision of methods used to determine low dissolved radiocesium concentrations in freshwater focused on repeatability and reproducibility have been performed. The results presented here therefore improve our understanding of the precision of such measurements.

Acknowledgements This study was supported partly by JSPS KAKENHI Grant Number 16H01791, a grant-in-aid from the Kurita Water and Environment Foundation (No. 16K004). We thank Gareth Thomas, Ph.D., from Edanz Group (www.edanzediting.com/ac) for editing a draft of this manuscript.

\section{Compliance with ethical standards}

Conflict of interest The authors declare that they have no conflict of interest.

Open Access This article is distributed under the terms of the Creative Commons Attribution 4.0 International License (http://creativeco mmons.org/licenses/by/4.0/), which permits unrestricted use, distribution, and reproduction in any medium, provided you give appropriate credit to the original author(s) and the source, provide a link to the Creative Commons license, and indicate if changes were made.

\section{References}

1. Ikeuchi Y (2015). In: Result report of the project in FY2014 for collecting data and developing migration models of the radioactive material distribution caused by Tokyo Electric Power Company Fukushima Daiichi Nuclear Power Station accident, part 1. Japan Atomic Energy Agency (in Japanese)

2. Oura Y, Ebihara M (2012) Radioactivity concentrations of ${ }^{131} \mathrm{I}$, ${ }^{134} \mathrm{Cs}$ and ${ }^{137} \mathrm{Cs}$ in river water in the Greater Tokyo Metropolitan 
area after the Fukushima Daiichi Nuclear Power Plant accident. Geochem J 46:303-309

3. Nagao S, Kanamori M, Ochiai S, Inoue M, Yamamoto M (2015) Migration behavior of ${ }^{134} \mathrm{Cs}$ and ${ }^{137} \mathrm{Cs}$ in the Niida River water in Fukushima Prefecture, Japan during 2011-2012. J Radioanal Nucl Chem 303:1617-1621

4. Sakaguchi A, Kadokura A, Steier P, Tanaka K, Takahashi Y, Chiga H, Matsushita A, Nakashima S, Onda Y (2012) Isotopic determination of $\mathrm{U}, \mathrm{Pu}$ and $\mathrm{Cs}$ in environmental waters following the Fukushima Daiichi Nuclear Power Plant accident. Geochem J 46:355-360

5. Ueda S, Hasegawa H, Kakiuchi H, Akata N, Ohtsuka U, Hisamatsu SI (2013) Fluvial discharges of radiocaesium from watersheds contaminated by the Fukushima Dai-ichi Nuclear Power Plant accident, Japan. J Environ Radioact 118:96-104

6. Nagao S, Kanamori M, Ochiai S, Suzuki K, Yamamoto M (2014) Dispersion of Cs-134 and Cs-137 in river waters from Fukushima and Gunma prefectures at nine months after the Fukushima Daiichi NPP accident. Prog Nucl Sci Technol 4:9-13

7. Yamashita R, Murakami M, Iwasaki Y, Shibayama N, Sueki K, Saha M, Mouri G, Lamxay S, Koibuchi Y, Takada H (2015) Temporal variation and source analysis of radiocesium in an urban river after the 2011 nuclear accident in Fukushima, Japan. J Water Environ Technol 13:179-194

8. Ochiai S, Ueda S, Hasegawa H, Kakiuchi H, Akata N, Ohtsuka Y, Hisamatsu SI (2015) Effects of radiocesium inventory on ${ }^{137} \mathrm{Cs}$ concentrations in river waters of Fukushima, Japan, under baseflow conditions. J Environ Radioact 144:86-95

9. Tsuji H, Yasutaka T, Kawabe Y, Onishi T, Komai T (2014) Distribution of dissolved and particulate radiocesium concentrations along rivers and the relations between radiocesium concentration and deposition after the nuclear power plant accident in Fukushima. Water Res 60:15-27

10. Yoshimura K, Onda Y, Sakaguchi A, Yamamoto M, Matsuura Y (2015) An extensive study of the concentrations of particulate/dissolved radiocaesium derived from the Fukushima Daiichi Nuclear Power Plant accident in various river systems and their relationship with catchment inventory. J Environ Radioact 139:370-378

11. Kameda Y, Yamaguchi H, Tamada M, Ohta S (2014) Establishment and evaluation of a novel rapid analysis method to measure trace levels of radioactive caesium in surface water by using absorbent disks for radioactive caesium with $\mathrm{NaI}$ scintillation counter (ADiCS). J Jpn Soc Water Environ 37:211-218 (in Japanese)

12. Nakanishi T, Sakuma K (2019) Trend of ${ }^{137}$ Cs concentration in river water in the medium term and future following the Fukushima nuclear accident. Chemosphere 215:272-279

13. Yoshikawa N, Obara H, Ogasa M, Miyazu S, Harada N, Nonaka $\mathrm{M}(2014){ }^{137} \mathrm{Cs}$ in irrigation water and its effect on paddy fields in Japan after the Fukushima nuclear accident. Sci Total Environ 481:252-259

14. Fujimura S, Yamaguchi H, Umehara T, Muto K (2013) Determination of radiocaesium concentration in irrigation water using sorbent disk. Radioisotopes 62:841-845 (in Japanese)

15. Shin M, Kubota T, Hamada K, Hitomi T, Ota T (2015) Dynamic analysis of radioactive cesium in decontaminated paddy fields. $\mathrm{J}$ Water Environ Technol 13:383-394

16. Ministry of Education, Culture, Sports, Science and Technology (1976) Radioactive cesium analysis method. Radioactivity measurement method series 3 (in Japanese)

17. Ministry of Education, Culture, Sports, Science and Technology (1982) Sample pre-treatment methods for instrumental analysis using germanium semiconductor detectors etc. Radioactivity measurement method series 13 (in Japanese)

18. Yasutaka T, Kawamoto T, Kawabe Y, Sato T, Sato M, Suzuki Y, Nakamura K, Konami T (2013) Rapid measurement of radiocesium in water using a Prussian blue impregnated nonwoven fabric. J Nucl Sci Technol 50:674-681

19. Tsuji H, Kondo Y, Suzuki Y, Yasutaka T (2014) Development of a method for rapid and simultaneous monitoring of particulate and dissolved radiocesium in water with nonwoven fabric cartridge filters. J Radioanal Nucl Chem 299:139-147

20. Yasutaka T, Tsuji T, Kondo Y, Suzuki Y (2013) Developemnt of rapid monitoring for dissolved radioactive cesium with a cartridge type of Prussian blue-impregnated nonwoven fabric. the Japan Society of Analytical Chemistry. Bunseki Kagaku 62:499-506 (in Japanese)

21. Yasutaka T, Tsuji H, Kondo Y, Suzuki Y, Takahashi A, Kawamoto $\mathrm{T}$ (2015) Rapid quantification of radiocesium dissolved in water by using nonwoven fabric cartridge filters impregnated with potassium zinc ferrocyanide. J Nucl Sci Technol 52:792-800

22. Yamaguchi H, Ota S, Tomita S (2012) Application of SPE-cesium disks to environmental monitoring of radiocesium in aqueous suspensions. In: SETAC Asia/Pacific conference 2012 proceedings. SETAC, Cleveland, Australia

23. Yamaguchi H, Ota S, Tsukada H (2014). Development of lowlevel-radiocesium concentration analysis system for irrigation water using solid phase extraction disks. In: 20th world congress of soil science proceedings, ICC, Jeju, Korea

24. Yasutaka T, Shin M, Onda Y, Shinano T, Hayashi S, Tsukada Y, Aono T, Iijima K, Eguchi S, Ohno K, Yoshida Y, Kamihigashi H, Kitamura S, Kubota T, Nogawa N, Yoshikawa N, Yamaguchi H, Sueki K, Tsuji H, Miyazu S, Okada Y, Kurihara M, Tarjan S, Matsunami H, Uchida S (2017) Comparison of values analyzed by deferent methods for concentrating low-level radio cesium dissolved in precipitation: evaporation, AMP, solid phase extraction disk, PB filter cartridge, ion-exchange, resins, Japan Society for Analytical Chemistry, the Japan Society of Analytical Chemistry. Bunseki Kagaku 66:299-307 (in Japanese)

25. Yasutaka T, Miyazu S, Kondo Y, Tsuji H, Arita K, Hayashi S, Takahashi A, Kawamoto T, Aoyama M (2016) Development of a copper-substituted, Prussian blue-impregnated, nonwoven cartridge filter to rapidly measure radiocesium concentration in seawater. J Nucl Sci Technol 53:1243-1250

26. Beals DM, Britt WG, Bibler JP, Brooks DA (1998) Radionuclide analysis using solid phase extraction disks. J Radioanal Nucl Chem 236:187-191

27. Beals D, Hofstetter K, Johnson VG, Patton GW, Seely D (2001) Development of field portable sampling and analysis systems. J Radioanal Nucl Chem 248:315-319

28. Aoyama M, Hirose K (2008) Radiometric determination of anthropogenic radionuclides in seawater. Radioact Environ 11:137-162

29. The technical manual of pre-treatment and analysis method of radiocesium in water for environmental monitoring, Review Committee of Technical Manual of Monitoring Methods of Radio Cesium in Water. https://unit.aist.go.jp/georesenv/informatio n/20150917/TechRep_Cs_Monitoring_Water.pdf\#search=\%27. Accessed 16 Jul 2019 (in Japanese)

30. Geurts R, Spooren J, Quaghebeur M, Broos K, Kenis C, Debaene L (2016) Round robin testing of a percolation column leaching procedure. Waste Manag 55:31-37

31. International Atomic Energy Agency (2013) IAEA analytical quality in nuclear applications series no. 29, emergency response proficiency test for Japanese laboratories: determination of selected radionuclides in water, soil, vegetation and aerosol filters. IAEA, Vienna

32. JIS Z 8405 (2018) Statistical methods for use in proficiency testing by interlaboratory comparisons. Japanese Industrial Standards, Tokyo (in Japanese)

Publisher's Note Springer Nature remains neutral with regard to jurisdictional claims in published maps and institutional affiliations. 


\section{Affiliations}

Momo Kurihara ${ }^{1,24} \cdot$ Tetsuo Yasutaka $^{1} \cdot$ Tatsuo Aono $^{2} \cdot$ Nobuo Ashikawa $^{3} \cdot$ Hiroyuki Ebina $^{4} \cdot$ Takeshi lijima $^{5}$. Kei Ishimaru ${ }^{6} \cdot$ Ramon Kanai $^{7} \cdot$ Zin'ichi Karube $^{8,25} \cdot$ Yae Konnai $^{9} \cdot$ Tomijiro Kubota $^{10}$ - Yuji Maehara ${ }^{11}$. Takeshi Maeyama ${ }^{12}$. Yusuke Okizawa ${ }^{13} \cdot$ Hiroaki Ota $^{14} \cdot$ Shigeyoshi Otosaka ${ }^{15,26}$. Aya Sakaguchi ${ }^{16}$. Hisaya Tagomori ${ }^{17} \cdot K^{2}$ eisuke Taniguchi ${ }^{18} \cdot$ Masatoshi Tomita $^{19} \cdot$ Hirofumi Tsukada ${ }^{20}$. Seiji Hayashi ${ }^{21}$. Sangyoon Lee ${ }^{22,27}$. Susumu Miyazu ${ }^{10,28} \cdot$ Moono Shin $^{22,29} \cdot$ Takahiro Nakanishi $^{23} \cdot$ Tatsuhiro Nishikiori ${ }^{22}$. Yuichi Onda ${ }^{16} \cdot$ Takuro Shinano $^{22,30} \cdot$ Hideki Tsuji $^{21}$

1 National Institute of Advanced Industrial Science and Technology, 1-1-1 Higashi, Tsukuba, Ibaraki 305-8567, Japan

2 National Institutes for Quantum and Radiological Science and Technology, Fukushima Branch Office QST, c/o FMU, Fukushima 960-1295, Japan

3 Kyushu Environmental Evaluation Association, 1-10-1 Higashi-ku, Matsukadai, Fukuoka, Fukuoka 183-0004, Japan

4 Japan Environment Science Co.,Ltd., 6 Takagi, Yamagata-City, Yamagata 990-2346, Japan

5 Environmental Control Center Co.,Ltd., 323-1 Shimoongata-machi, Hachioji, Tokyo 192-0154, Japan

6 IDEA Consultants, Inc., 3-15-1 Komazawa, Setagaya-ku, Tokyo 154-8585, Japan

7 Tokyo Power Technology Ltd., 2-3-6 Onodai, Midori-ku, Chiba, Chiba 267-0056, Japan

8 Center for Water Environment Studies, Ibaraki University, 1375 Ohu, Itako, Ibaraki 311-2402, Japan

9 Nippon Chemical Environment Center Co.,Ltd., 2-25 Matsuki-cho, Koriyama, Fukushima 963-8812, Japan

10 Institute for Rural Engineering, National Agriculture and Food Research Organization, 2-1-6 Kannondai, Tsukuba, Ibaraki 305-8609, Japan

11 Chugai Technos Corporation, 2-2-16 Ohnodai Midori-ku, Chiba 267-0056, Japan

12 Japan Chemical Analysis Center, 295-3, Sanno-cho, Inage-ku, Chiba City, Chiba 263-0002, Japan

13 Kankyo Bunseki Kenkyusho Co.,Ltd., 22-2 Higashihama, Fukushima, Fukushima 960-8132, Japan

14 Suncoh Environmental Research Center Co.,Ltd., 1-4-1 Tamagawa, Chofu, Tokyo 182-0025, Japan

15 Japan Atomic Energy Agency, 2-4 Shirakata, Tokai, Ibaraki 319-1195, Japan

16 Center for Research in Isotopes and Environmental Dynamics, University of Tsukuba, 1-1-1 Tenno-dai, Tsukuba, Ibaraki 305-8572, Japan

17 Japan Atomic Energy Agency, 10-2 Fukasaku, Miharu, Fukushima 963-7700, Japan
18 Fukushima Prefectural Centre for Environmental Creation, 10-2 Fukasau, Miharu, Fukushima 963-7700, Japan

19 KANSO CO.,LTD., 3-1-1 Higashikuraji, Katano, Osaka 576-0061, Japan

20 Institute of Environmental Radioactivity, Fukushima University, 1 Kanayagawa, Fukushima, Fukushima 960-1296, Japan

21 National Institute for Environmental Studies, 10-2 Fukasaku, Miharu, Fukushima 963-7700, Japan

22 Tohoku Agricultural Research Center, National Agriculture and Food Research Organization, 50 Harajuku-minami, Arai, Fukushima, Fukushima 960-2156, Japan

23 Japan Atomic Energy Agency, 45-169 Kaihama-sukakeba, Haramachi, Minamisoma, Fukushima 975-0036, Japan

24 Present Address: National Institutes for Quantum and Radiological Science and Technology, 4-9-1 Anagawa, Inage-ku, Chiba, Chiba 263-8555, Japan

25 Present Address: Department of Biotechnology and Chemistry, Faculty of Engineering, Kindai University, 1 Takayaumenobe, Higashihiroshima, Hiroshima 739-2116, Japan

26 Present Address: Atmosphere and Ocean Research Institute, The University of Tokyo, 5-1-5 Kashiwanoha, Kashiwa, Chiba 277-8564, Japan

27 Present Address: Western Region Agricultural Research Center, National Agriculture and Food Research Organization, 2575 Ikano-cho, Zentsuji-shi, Kagawa 765-0053, Japan

28 Present Address: Faculty of Agriculture, Niigata University, Karashi 2-no-cho, Nishi-Ku, Niigata 950-2181, Japan

29 Present Address: Faculty of Food and Agricultural Sciences, Fukushima University, 1 Kanayagawa, Fukushima, Fukushima 960-1296, Japan

30 Present Address: Laboratory of Plant Nutrition, Research Faculty of Agriculture, Hokkaido University, N9 W9, Kitaku, Sapporo, Hokkaido 060-8589, Japan 